\title{
Normal Frequencies of a Simple Cubic Lattice. III
}

\author{
J. O. HALFORD \\ Chemistry Department, University of Michigan, Ann Arbor, Michigan
}

(Received January 29, 1952)

\begin{abstract}
For a simple cubic crystal, with forces resisting displacement along and perpendicular to the connecting line between closest neighbors, the secular equation for the internal motions is rigorously broken down to ultimate factors of the form

$$
\nu^{2}=\nu_{x}^{2}+\alpha\left(\nu_{y}^{2}+\nu_{x}^{2}\right),
$$

in which $\nu_{x}$, etc., are the normal frequencies of the one-dimensional crystal.

The corresponding frequency distribution shows no indication of the "infinities" found by Montroll for a model with central forces between first and second neighbors.
\end{abstract}

$\mathrm{N}$ the first paper of this series ${ }^{1}$ it was shown that the $n$th frequency of a one-dimensional crystal can be accurately expressed by the equation

$$
\nu_{n}=\left(k / \pi^{2} m\right)^{\frac{1}{2}} \sin (n \pi / 2 N) ; 1<n<N-1,
$$

in which $k$ is the force for unit relative displacement of neighbors of uniform mass $m, n$ is an integer, and $N$ is the number of particles in the chain.

In the second section ${ }^{1}$ it was proposed empirically that the frequencies of an isotropic three-dimensional array of uniform masses might be given by the equation

$$
\nu^{2}=\nu_{x}^{2}+\alpha\left(\nu_{y}{ }^{2}+\nu_{z}^{2}\right)
$$

as a simplified approximation to the factored secular equation. Here $\nu_{x}$, etc., are sets of solutions of Eq. (1). While Eq. (2) should lead to a frequency distribution accurate enough for a variety of models, it is probably the exact solution for only one model. Because of the empirical origin of Eq. (2), the description of the corresponding model was not originally obvious, but a search for the model was undertaken for the purpose of providing the equation with as good a physical foundation as possible.

It develops that Eq. (2) is the exact factored secular equation for the simple cubic crystal studied by Houston, ${ }^{2}$ in which the restoring forces are $k$ for unit relative displacement of closest neighbors along their connecting line and $k \alpha$ for unit displacement perpendicular to the connecting line. While this model is a somewhat simplified representation of a real system, it could lead to a good approximation to a generally useful frequency distribution.

The operations which break down the secular equation into factors of the form of Eq. (2) are simple and straightforward. In the first place, motion along each of the three Cartesian coordinate directions parallel to the edges of a rectangular parallelipiped of particles is independent of the other directions. Consequently the secular equation is automatically factored into three equivalent parts. Secondly, each of these parts can be factored to the form of Eq. (2) by repeated use of the

\footnotetext{
${ }^{1}$ J. O. Halford, J. Chem. Phys. 19, 1375 (1951).

2 W. V. Houston, Revs. Modern Phys. 20, 161 (1948).
}

factoring operations implied in the derivation of Eq. (1) for the one-dimensional crystal.

The solution of the problem for a cube with three particles on the edge will be developed. The extension of the secular determinant to a larger model will be described and it will be evident, from the method of factoring, that the factors found for the twenty-sevenparticle model, with appropriate interpretation of the symbols, are the rigorous factors for the general rectangular parallelipiped of dimensions $N_{1} \times N_{2} \times N_{3}$.

For the $3 \times 3 \times 3$ cube, the particles in the successive planes perpendicular to the direction of motion are numbered as in the following illustration:

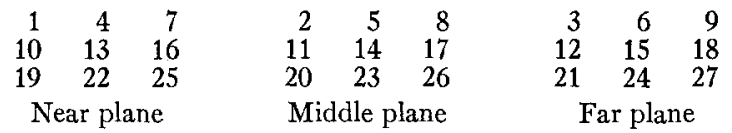

and the elements are introduced into the secular determinant in the corresponding order. For example, the elements in the eighth row and column are made up from the energy terms involving $x_{8}$ and $\dot{x}_{8}$ for particle number eight.

With the substitution $u=\left(4 \pi^{2} \nu^{2} m / k\right)-2$, the secular determinant takes the form shown in Eq. (3). Only half the determinant, as far as the secondary diagonal (broken line), is shown. The rest of the determinant is obtained by reflection through the secondary diagonal. Each successive set of three rows or columns is associated with a row of masses aligned in the direction motion, while each set of nine is connected with a plane of particles parallel to a face of the model and to the direction of motion. The determinant is divided into $3 \times 3$ blocks of which those on the diagonal belong specifically to individual rows. The off-diagonal blocks show the cross-linking between one row and another. The $9 \times 9$ portions are associated in an analogous way with planes parallel to a face of the model.

The determinant for the general $N_{1} \times N_{2} \times N_{3}$ parallelipiped can be described with the aid of Eq. (3). For the elements in the main diagonal, extension in the direction of motion involves extending the $3 \times 3$ blocks to $N_{1} \times N_{1}$ through repetition of the middle element of each block. Extension in the second dimension requires enlarging the $9 \times 9$ blocks to $N_{1} N_{2} \times N_{1} N_{2}$ through 
repetition of the middle $N_{1} \times N_{1}$ block of each set of three. For the third dimension, the extension involves repetition of the middle $N_{1} N_{2} \times N_{1} N_{2}$ block until the dimensions of the whole determinant are $N_{1} N_{2} N_{3}$ $\times N_{1} N_{2} N_{3}$. The rows of unit cross-product coefficients parallel to the main diagonal are unbroken within the $N_{1} \times N_{1}$ blocks but are interrupted as in Eq. (3) at the end of each such block.

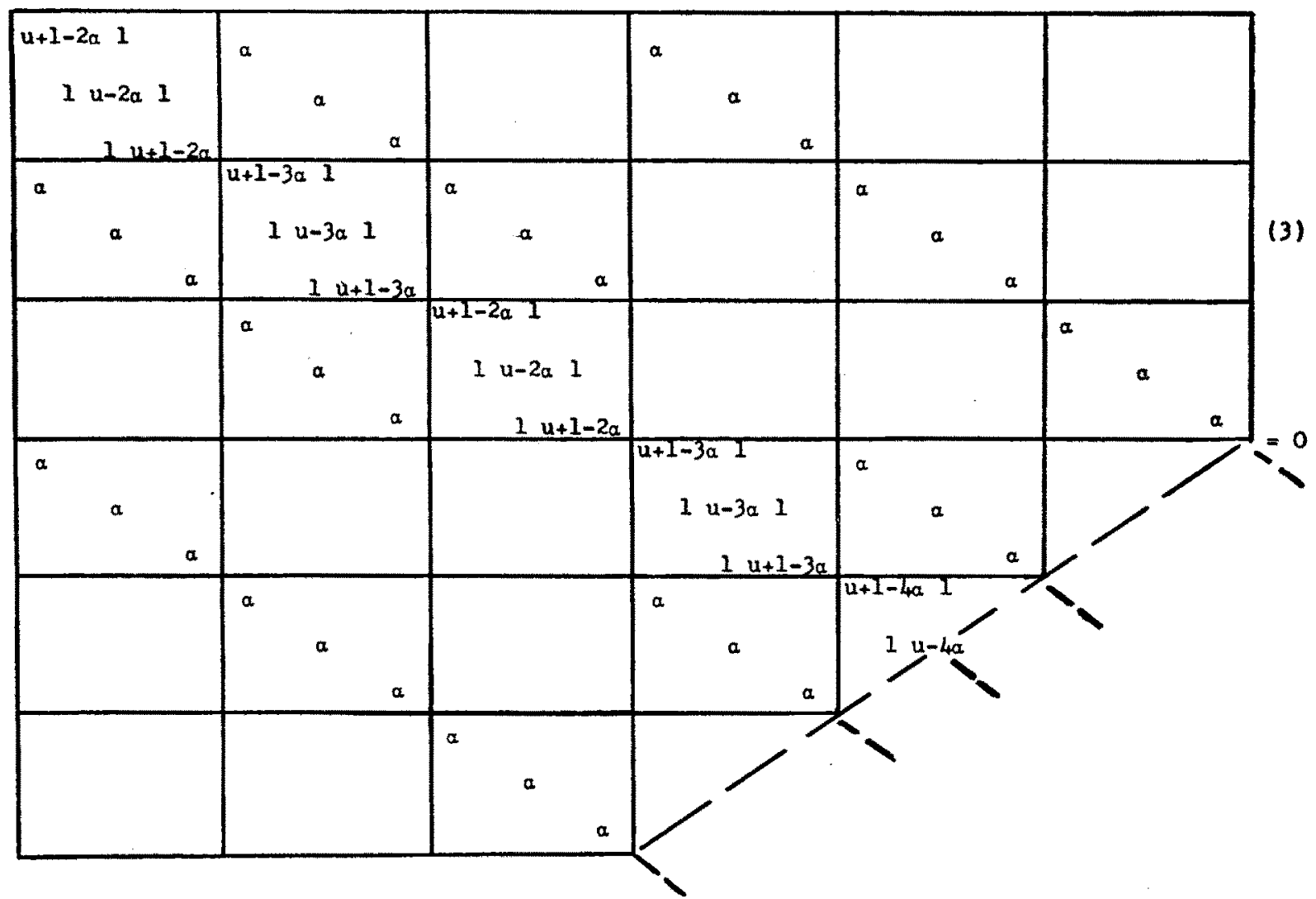

In a similar way the inner rows of alphas are unbroken within each $N_{1} N_{2} \times N_{1} N_{2}$ square but $N_{1}$ alphas are missing at the ends of each of these squares. The outer rows of alphas extend without interruption through the whole determinant.

The factoring of Eq. (3) (or the general secular equation) is accomplished by using the secular determinant for the one-dimensional crystal as a key. For three particles in a line the secular equation, with $u$ defined as above, has the form

$$
\left|\begin{array}{ccc}
u+1 & 1 & \\
1 & u & 1 \\
& 1 & u+1
\end{array}\right|=0 .
$$

The roots are known, by Eq. (1), and this implies that the determinant has been diagonalized, by the use of linear combinations of the corresponding elements of the rows and columns, to yield the equation

$$
\left(u-u_{1}\right)\left(u-u_{2}\right)\left(u-u_{3}\right)=0
$$

or, in the general case for $N$ particles,

$$
\left(u-u_{1}\right) \cdots\left(u-u_{i}\right) \cdots\left(u-u_{N}\right)=0 .
$$

It becomes evident that the application of the same factoring operations, in the same order, will diagonalize the more general determinant, $F$, as shown in Eq. (7).

$$
\begin{gathered}
F \equiv\left|\begin{array}{ccc}
u+\beta+\gamma & \beta & \\
\beta & u+\gamma & \beta \\
\beta & u+\beta+\gamma
\end{array}\right| \\
\quad=\left(u+\gamma-\beta u_{1}\right)\left(u+\gamma-\beta u_{2}\right)\left(u+\gamma-\beta u_{3}\right)=0 .
\end{gathered}
$$

By dividing each element by $\beta$ and substituting $v=(u+\gamma) / \beta$, the determinant takes the form of Eq. (4), and has the factored form $\left(v-u_{1}\right)\left(v-u_{2}\right)\left(v-u_{3}\right)=0$, which, upon reversing the substitution and multiplying each factor by $\beta$, reverts to Eq. (7). Obviously the constants $\beta$ and $\gamma$ can have any values, including zero.

If this set of factoring operations is applied, in Eq. (3), to each $3 \times 3$ square along the diagonal, every other $3 \times 3$ square will automatically be subjected to the same operations, and, since all squares have the form of Eq. (7), they will all be diagonalized, and the particular root $u_{i}$ will appear in the same position in all squares in which it occurs. By rearranging the order of the rows and columns, the determinant becomes factored into 3 (in the general case $N_{1}$ ) exactly similar 
determinants, each of which contains only one of the roots $u_{i}$, as follows:

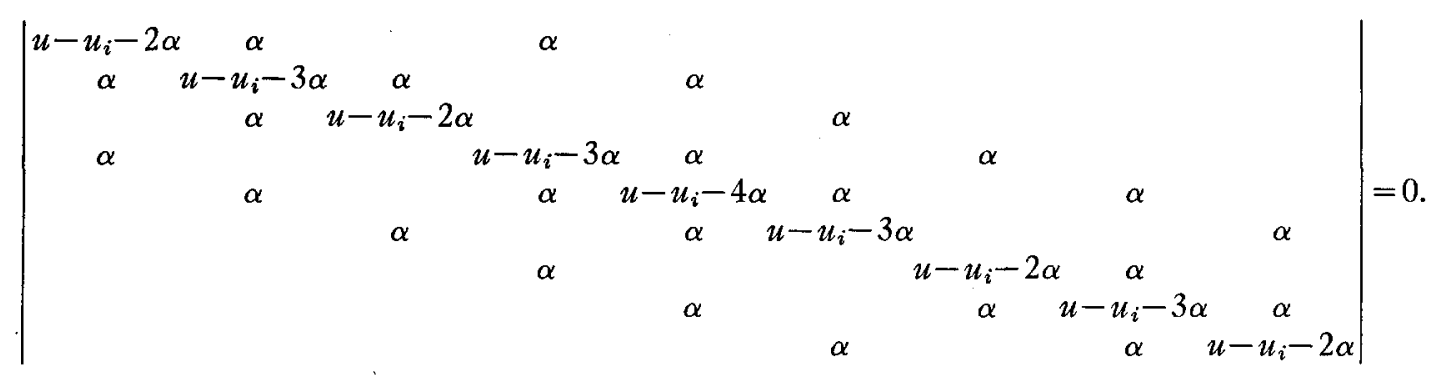

In Eq. (8), each $3 \times 3$ (in the general case $N_{2} \times N_{2}$ ) square again has the same form as the determinant $F$ of Eq. (7). For example, in the first square on the diagonal, substitute $\gamma=-\left(u_{i}+3 \alpha\right)$ and $\beta=\alpha$ to show the equivalence. Consequently, repetition of the previously used operations will break down Eq. (8) into 3 (in the general case $N_{2}$ ) factors of the form of Eq. (9), each containing one root $u_{j}$ of the linear crystal equation.

$$
\left|\begin{array}{ccc}
u-u_{i}-3 \alpha-\alpha u_{j} & \alpha & \\
\alpha & u-u_{i}-4 \alpha-\alpha u_{j} & \alpha \\
\alpha & u-u_{i}-3 \alpha-\alpha u_{j}
\end{array}\right|=0 .
$$

Equation (9) again has the form of Eq. (7), and the ultimate factors, 3 (or $N_{3}$ ) in number, are

$$
u-u_{i}-4 \alpha-\alpha\left(u_{j}+u_{k}\right)=0 .
$$

It should be clear that this is the general factored form of the secular equation for a parallelipiped of dimensions $N_{1} \times N_{2} \times N_{3}$. Since $u=\left(4 \pi^{2} \nu^{2} m / k\right)-2$ and the roots $u_{i}$ etc. can be similarly expressed, it follows that

$$
\nu^{2}=\nu_{i}^{2}+\alpha\left(\nu_{j}^{2}+\nu_{k}^{2}\right),
$$

which is exactly the same as Eq. (2), proposed empirically in the earlier paper.

It is interesting that, in contrast to common experience in this kind of study, it has not been necessary to neglect end corrections. Equation (11) is the exact factored form of the secular equation for the model as defined, without any approximations whatever. Consequently, any inadequacy of the result is the fault of the potential energy function, but is not attributable to the derivation. By comparison with the probable properties of real crystals, the model is clearly oversimplified. For example, it does not provide for the rotations of the entire parallelipiped, since only three zero roots, obviously translations, can be obtained from Eq. (11) in its three permissible permutations.

Nevertheless the cubic model employed here seems far closer to physical reality than Debye's continuous model, and should therefore lead to a frequency distribution which is more generally useful than the Debye function. The distribution curve will contain four discontinuities, but will show no indication of the "infinities" found by Montroll ${ }^{3}$ for a closely related model. It would therefore be a mistake to claim, at present, that such "infinities" are a necessary feature of the distribution curve for two- and three-dimensional systems.

Unfortunately, it has not proved to be possible to check Montroll's distribution independently by factoring the secular equation for his model by the methods used in the present paper. Approximations can be obtained, however, if the coefficients of certain crossproducts are neglected or reversed in sign. In each approximation the resulting distribution curve resembles the one prescribed by Eq. (11) more closely than the Montroll curve. It seems, therefore, that Montroll's "infinities" are quite sensitive to the detail of the potential energy function, since they disappear when secondary changes are made in this function. This tends to support the hypothesis that such peaks are not a necessary feature of the distribution curve.

At some future date an attempt will be made to set up and test against thermal data a usable continuous distribution function with an acceptable limiting slope at zero frequency, which will not deviate too far from the distributions prescribed by Eq. (11) and by Montroll's studies.

${ }^{3}$ E. W. Montroll, J. Chem. Phys. 15, 575 (1947). 